\title{
Robustness of Classifiers to Changing Environments
}

\author{
Houman Abbasian ${ }^{1}$, Chris Drummond ${ }^{2}$, Nathalie Japkowicz ${ }^{1}$ and Stan \\ Matwin $^{1,3}$ \\ 1 School of Information Technology and Engineering, \\ University of Ottawa \\ Ottawa, Ontario, Canada, K1N 6N5 \\ habba057@uottawa.ca,nat@site.uottawa.ca, stan@site.uottawa.ca \\ 2 Institute for Information Technology, \\ National Research Council of Canada, \\ Ottawa, Ontario, Canada, K1A 0R6 \\ Chris.Drummond@nrc-cnrc.gc.ca \\ 3 Institute of Computer Science, \\ Polish Academy of Sciences, \\ Warsaw, Poland
}

\begin{abstract}
In this paper, we test some of the most commonly used classifiers to identify which ones are the most robust to changing environments. The environment may change over time due to some contextual or definitional changes. The environment may change with location. It would be surprising if the performance of common classifiers did not degrade with these changes. The question, we address here, is whether or not some types of classifier are inherently more immune than others to these effects. In this study, we simulate the changing of environment by reducing the influence on the class of the most significant attributes. Based on our analysis, K-Nearest Neighbor and Artificial Neural Networks are the most robust learners, ensemble algorithms are somewhat robust, whereas Naive Bayes, Logistic Regression and particularly Decision Trees are the most affected.
\end{abstract}

Key words: classifier evaluation, changing environments, classifier robustness

\section{Introduction}

In this paper, we test some of the most popular, and commonly used, classifiers to identify which are the most robust to changing environments. The environment is the state of the world when the data set is first collected. Essentially, the data set is a single snapshot of the world, capturing its state. This state may change over time, as time passes from first deployment of the classifier in the field. This state may even change in the time from when the classifier is trained to when it is deployed. This state may change with situation: different locations of the same application will have appreciable differences. It would be surprising if the 
performance of a classifier did not degrade with these changes. The question we address here is whether or not some classifiers are inherently more immune than others to such changes. Although there has been considerable work on the effect of changing the distribution of the class attribute on classifier performance $[8$, $9,5,4]$, only more recently has research looked at the effect of changes in the distribution of other attributes $[1,7]$. This is an area of growing importance, as evidenced by a recent book on the topic [11].

For the purposes of discussion, let us divide the remaining attributes into three types: conditions, outcome and context. This division captures the causal structure of many problems. Conditions cause the class. This is particularly apparent in medicine where they are called risk factors. A change in risk factor causes a change in the frequency of the disease. For example, the population, in most western countries, is aging and putting on weight yet smoking less and eating more carefully. To what extent will a classifier, say predicting heart problems, be impervious to such changes? The class causes the outcomes. In medicine these would be the symptoms. We might expect, however, that the symptoms of a disease would remain constant. Nevertheless, we could see the concept change over time, i.e. concept drift [14], e.g. as our understanding of the implications of the disease is clarified. Context causes changes in other attributes but not the class $[13,12]$. For example, the increase in the prevalence of other diseases in the population may mask the symptoms of a heart attack, and therefore degrade any classifier's performance.

We discuss here the changes in the influence of a particular attribute on the class, which can be measured by information gain. We think this is most likely to occur with changes in conditions, although changes in context and outcome may also have an effect. A good example of changes in risk factors, and with them changes in the influence of attributes, is type- 2 diabetes. This disease, originally called adult onset diabetes, has lately turned up in alarming numbers in children. Age might have originally been a strongly predictive attribute but it is no longer. Smoking and lung cancer are strongly related, but as more people quit smoking the influence of other factors will begin to dominate. Simply put, the predictive power of attributes will change with time.

This research is related to previous work by one of the authors that also addressed the robustness of classifiers [2]. However, we would contend that the issue of the robustness of classifiers is a significant and growing area and many aspects warrant investigation. This paper differs in the type of change investigated - the previous work changed the distribution of the data in the test set while in this work we change the influence of the most significant attributes - and the simulation of change based on real data sets rather than an artificial one. More importantly, it differs in that it focuses on algorithms that learn more complex representations. We surmise that algorithms that produce classifiers reliant on a large number of attributes should be more robust to such changes than the ones reliant on a small number. This is, in general, the conclusion we draw from our experiments although there are subtleties which we discuss later in the paper. Based on our analysis, K-Nearest Neighbor and Artificial Neural Networks are 
the most robust learners, ensemble algorithms are somewhat robust, whereas Logistic Regression, Naive Bayes and particularly Decision Trees fare badly.

The remainder of this paper is organized as follows. Section 2 describes our testing methodology and Section 3 the experimental results. Section 4 is a discussion of why some learners are more robust than others and suggestions for future work.

\section{Testing Methodology}

Our experiments are designed to answer two questions: (a) Which learners are the most robust to changing environments? (b) Does the reliance on a large number of attributes make a classifier more robust?

To empirically answer the above questions, we selected a good variety of data sets. We used 6 data sets from the UCI repository [3]: Adult, Letter, Nursery, Connect-4, Breast Cancer and Vote. The number of classes ranges from 2 to 26; the number of instances from 569 to $67557^{1}$; the number of attributes from 8 to 42 and the types are both continuous and categorical. All algorithms come from the Weka data mining and machine learning tool [16]. The classifiers produced range from those that are reliant on a small number of attributes such as the decision tree algorithm J48, through those of ensemble algorithms that rely on a larger number - Random Forest, Bagging, AdaBoost - to ones that use all attributes such as Naive Bayes, Logistic Regression, Artificial Neural Networks, K-Nearest Neighbor. In this study, the five most significant attributes are determined using the "Gain Ratio Attribute Evaluator" of Weka. For all of the experiments, we changed the influence of the attributes by changing its information gain ratio, as discussed in the next section. We train each algorithm using the original data and test the classifier on data where the influence of different attributes on the class is progressively decreased.

\subsection{Changing an Attribute's Influence}

One measure for the influence of an attribute on the class is information gain, defined as the expected reduction in entropy caused by partitioning the examples according to that attribute. To decrease the influence of an attribute, we reduce the information gain by adding noise to that attribute. Equation 1 shows the information gain of attribute $A$ relative to a collection of examples with class labels $C$.

$$
\begin{aligned}
\operatorname{Gain}(C, A) & =\operatorname{Entropy}(C)-\sum_{v \in \operatorname{Values}(A)} \frac{\left|C_{v}\right|}{|C|} \operatorname{Entropy}\left(C_{v}\right) \\
\operatorname{Entropy}\left(C_{v}\right) & =-\sum_{i=1}^{c} p_{i} \log _{2} p_{i}
\end{aligned}
$$

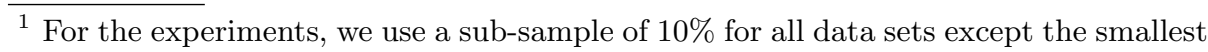


$\operatorname{Values}(A)$ is the set of all possible values for attribute $A ; C_{v}$ is the subset of $C$ for which attribute $A$ has value $v ; p_{i}$ is the proportion of $C_{v}$ belonging to class $i$. In equation 1 , adding noise to attributes does not change $C$ but will change $C_{v}$. The noise randomly changes attribute values of selected instances. So, the proportion $p_{i}$ of each class associated with a particular value will move closer to the proportion of classes in the whole data set. Therefore, Entropy $\left(C_{v}\right)$ moves towards Entropy $(C)$ resulting in a lower $\operatorname{Gain}(C, A)$.

In this paper, we used a slightly modified version of information gain called gain ratio. ${ }^{2}$ This measure includes a term called split information which is sensitive to how broadly the attribute splits the data and defined by equation 2 . By adding noise to attributes, the $\left|C_{i}\right|$ will move closer to each other. Therefore SplitInfo in equation 2 will become larger and as a result the gain ratio will be lower.

$$
\begin{aligned}
\operatorname{GainRatio}(C, A) & =\frac{\operatorname{Gain}(C, A)}{\operatorname{SplitInfo}(C, A)} \\
\operatorname{SplitInfo}(C, A) & =-\sum_{i=1}^{c} \frac{\left|C_{i}\right|}{|C|} \log _{2} \frac{\left|C_{i}\right|}{|C|}
\end{aligned}
$$

In the following, rather than record the actual gain ratio, we record change level. This is the percentage of test instances whose attribute values have been changed. Thus $100 \%$ means all values have been altered. If the attribute is nominal, the existing value is replaced by one randomly selected from the remaining values of that attribute. If the attribute is numeric, it is replaced by a randomly generated number drawn from the uniform distribution, spanning the maximal and minimal values of that attribute.

\section{Experimental Results}

In this section, we first determine which attributes have the largest effect on performance. We then compare classifiers to see how they fare as the influence of the attributes decreased.

\subsection{The Influence of each Attribute on Accuracy}

Some attributes will have a strong influence on the class; others will have a much weaker influence. To determine the strength, we found the five most influential attributes, based on the information gain ratio, for each data set. We then used 10-fold cross validation to calculate the performance of each learner, averaged over all change levels. Table 1 shows the different degrees of influence that the attributes have on the class. The $*$ in this table indicates that, using a paired t-test with an alpha of 0.05 , the accuracy of the learner for corrupted data is significantly different from that for the original data.

\footnotetext{
${ }^{2}$ The use of information gain as a way of selecting significant attributes has a long history in machine learning. So does this modified version, which is used to select attributes in $\mathrm{C} 4.5[10]$
} 
Table 1. Accuracy with decreasing the gain ratio

\begin{tabular}{|c|c|c|c|c|c|c|c|c|c|}
\hline Data set & Attribute & $\mathrm{J} 48$ & $\mathrm{RF}$ & NB & BG & $\mathrm{ADB}$ & KNN & LR & ANN \\
\hline \multirow{6}{*}{ Adult } & 11 & $53.22^{*}$ & $70.19^{*}$ & $49.2^{*}$ & $74.4^{*}$ & $73.44^{*}$ & 80.35 & $57.09^{*}$ & 80.25 \\
\hline & 12 & 83.3 & 82.52 & 68.7 & 82.6 & 83.22 & 80.75 & 81.83 & 80.47 \\
\hline & 6 & 82.04 & 82.32 & 81.4 & 82.7 & 80.62 & 80.18 & 82.1 & 80.37 \\
\hline & 8 & 83.17 & 81.54 & 81.5 & 78.3 & 83.22 & 80.52 & 83.05 & 80.73 \\
\hline & 10 & 83.87 & 82.63 & 81.6 & 82.7 & 83.22 & 80.7 & 83.43 & 81.13 \\
\hline & Original Data & 82.64 & 82.48 & 82 & 82.3 & 83.37 & 80.7 & 83.5 & 80.87 \\
\hline \multirow{6}{*}{ Letter } & 14 & $49.05^{*}$ & $63.69^{*}$ & $49.2^{*}$ & $55.5^{*}$ & 6.749 & $60.52^{*}$ & 51.35* & $21.21^{*}$ \\
\hline & 13 & $61.88^{*}$ & $73.7^{*}$ & $54.1^{*}$ & $70^{*}$ & 6.749 & $66.71^{*}$ & $61.84^{*}$ & $24.27^{*}$ \\
\hline & 11 & $49.56^{*}$ & $75.81^{*}$ & $52.7^{*}$ & $67.9^{*}$ & 6.749 & $68.94^{*}$ & $58.4^{*}$ & $25.2^{*}$ \\
\hline & 7 & $63.63^{*}$ & $73.03^{*}$ & $54.7^{*}$ & $67^{*}$ & 6.749 & $70.66^{*}$ & $65.98^{*}$ & $26.01^{*}$ \\
\hline & 12 & $59.95^{*}$ & $72.42^{*}$ & $50.8^{*}$ & $63.5^{*}$ & 6.749 & $64.15^{*}$ & $60.08^{*}$ & $27.1^{*}$ \\
\hline & Original Data & 70.94 & 80.46 & 62 & 76.4 & 6.916 & 75.34 & 74.82 & 29.48 \\
\hline \multirow{6}{*}{ Nursery } & 8 & $63.38^{*}$ & $65.31^{*}$ & $64.4^{*}$ & $63.8^{*}$ & $46.68^{*}$ & $63.06^{*}$ & $64.85^{*}$ & $65.92^{*}$ \\
\hline & 2 & $79.86^{*}$ & $79.98^{*}$ & $77.1^{*}$ & $78.7^{*}$ & 65.89 & $79.23 *$ & $80.3^{*}$ & $80.35^{*}$ \\
\hline & 1 & $84.33^{*}$ & $85.06^{*}$ & $84.7^{*}$ & $84^{*}$ & 65.89 & $82.46^{*}$ & $84.92 *$ & $85.5^{*}$ \\
\hline & 7 & 88.41 & 90.15 & 87.9 & 88.5 & 65.89 & 86.67 & 89.18 & 89.92 \\
\hline & 5 & 88.9 & 90.26 & 88.1 & 88 & 65.89 & 85.68 & 89.99 & 90.32 \\
\hline & Original Data & 90.02 & 91.66 & 88.6 & 88.5 & 65.51 & 87.89 & 91.66 & 91.72 \\
\hline \multirow{6}{*}{ Connect-4 } & 36 & 79.8 & 80.63 & $58.8^{*}$ & 81.4 & 72.79 & 78.13 & 58.34 & 83.61 \\
\hline & 35 & 79.8 & 80.62 & $57.9^{*}$ & 81.4 & 72.79 & 78.13 & 64.47 & 83.42 \\
\hline & 21 & $70.59^{*}$ & $76.3^{*}$ & $65.1^{*}$ & $72.6^{*}$ & $69.1^{*}$ & 76.26 & $54.12^{*}$ & $76.86^{*}$ \\
\hline & 18 & 79.8 & 80.63 & $59.8^{*}$ & 81.4 & 72.79 & 78.12 & 65.24 & 83.57 \\
\hline & 41 & 79.8 & 80.64 & $58.1^{*}$ & 81.4 & 72.79 & 78.13 & 64.89 & 83.49 \\
\hline & Original Data & 80.02 & 80.33 & 71.7 & 81.8 & 73.25 & 78.05 & 65.11 & 82.02 \\
\hline \multirow{6}{*}{ Breast Cancer } & 5 & $65.71^{*}$ & (68.61 & $\overline{72.3}$ & 699.8 & 73.93 & 72.95 & 71.13 & 74.74 \\
\hline & 4 & 73.24 & 70.06 & 71.8 & 69.2 & 73.07 & 73.94 & 71.04 & 74.72 \\
\hline & 6 & 70.95 & 68.24 & 73.6 & 69.2 & 69.41 & 72.66 & 70.96 & 72.79 \\
\hline & 9 & 74 & 70.11 & 73.7 & 70.2 & 74.78 & 73.82 & 72.02 & 75.57 \\
\hline & 3 & 74.07 & 67.77 & 73.7 & 72.1 & 74.98 & 74.8 & 71.52 & 75.74 \\
\hline & Original Data & 76.44 & 68.6 & 74.6 & 70.4 & 75.91 & 75.87 & 71.42 & 75.28 \\
\hline \multirow{6}{*}{ Vote } & $\overline{4}$ & $67.91^{*}$ & $72.12^{*}$ & $85.7^{*}$ & $67.7^{*}$ & $73.47^{*}$ & 89.91 & $78.33^{*}$ & $74.87^{*}$ \\
\hline & 3 & 96.07 & 95.35 & 90.5 & 96.7 & 96.26 & 91.04 & 88.54 & 94.44 \\
\hline & 5 & 96.07 & 95.51 & 89.7 & 96.7 & 96.7 & 92.03 & 83.36 & 95.13 \\
\hline & 12 & 95.94 & 96.45 & 90.1 & 96.5 & 96.33 & 92.06 & 91.71 & 95.25 \\
\hline & 14 & 96.1 & 95.64 & 89.7 & 96.7 & 96.6 & 91.59 & 90.85 & 95.72 \\
\hline & Original Data & 96.11 & 95.17 & 89.6 & 96.4 & 96.11 & 92.15 & 92.84 & 94.69 \\
\hline
\end{tabular}

For the Adult, Vote and Connect-4 data sets, only one attribute has a substantial influence. For others, more attributes are influential: three attributes for Nursery, and all five for the Letter data set. For the Breast Cancer data set, only for the Decision Tree is there a statistically significant difference and then only for the first attribute. However, as the percentage of positive samples is $30 \%$, 
the accuracy of all algorithms on the original data is very close to $70 \%$, that of the default classifier. Thus corrupting the attributes has little impact. We will drop this data set from further consideration.

For the Letter data set, AdaBoost uses a Decision Stump as its base classifier. The performance of a Decision Stump, on this data set, is close to that of default classifier, so corrupting the attributes has little effect on its accuracy. The performance of the Neural network is also low, yet higher than that of AdaBoost and the change level does affect its performance significantly. The surprising point for connect- 4 data set is that the first and the second most influential attributes do not have an impact on the accuracy that is statistically significant for any learner. However, the third most influential attribute does. Exactly why this occurs will need further investigation.

\subsection{Ranking the Algorithms}

For each learner and each data set, we use the attributes that significantly affect the accuracy of the learner. First, we trained each learner using the original data set. We then tested it on data where the influence of the attributes has been decreased. We decreased the influence of each attribute using different change levels $(20 \%, 40 \%, 60 \%, 80 \%$, and $100 \%)$. The performance of each learner was averaged over all attributes for each change level. It should be noted that in Table 2 smaller is better, i.e. there is less change in performance. The best value in each row is in bold type. The average value of the best performing learner is underlined if it is significantly better than the value from the second best learner, using a paired t-test with a significance level of 0.05 .

Let us go through the results of Table 2 by data set:

Adult The performances on clean and corrupted data are essentially indistinguishable for Nearest Neighbor and the Neural Network. The small negative numbers are likely caused by random errors. So, we conclude, on average across all change levels, the Neural Network and Nearest Neighbor are the most robust learners.

Letter AdaBoost appears initially to be the most robust but, as explained earlier, the accuracy of AdaBoost on the original data set is very low and changing attributes has little effect. Excluding AdaBoost, Neural Network is the most robust at all levels. On average across all change levels, the Neural Network is the most robust learner and Random Forest is second.

Nursery Bagging is the most robust at lower change levels, while Nearest Neighbor is the best at higher levels. In addition, on average Nearest Neighbor is the most robust method but, using the t-test, it is not significantly different from the second best learner, Bagging.

Connect-4 Nearest Neighbor is the best learning algorithm at all change levels. On average across all levels, Nearest Neighbor is the best learner and AdaBoost the second best.

Vote Nearest Neighbor is the best model at all change levels. On average across all levels, Naive Bayes is the second best. 
Table 2. The impact of change level on the difference in performance

\begin{tabular}{|c|c|c|c|c|c|c|c|c|c|}
\hline Data set & Change Level & J48 & $\mathrm{RF}$ & NB & BG & $\overline{\mathrm{ADB}}$ & KNN & $\overline{\mathrm{LR}}$ & $\overline{\mathrm{ANN}}$ \\
\hline \multirow{6}{*}{ Adult } & $20 \%$ & 9.25 & 3.83 & 11.29 & 2.42 & 3.56 & -0.35 & 8.04 & -0.86 \\
\hline & $40 \%$ & 18.94 & 8.12 & 22.35 & 5.42 & 7.30 & -0.55 & 17.88 & -0.36 \\
\hline & $60 \%$ & 29.25 & 12.90 & 31.43 & 7.61 & 10.04 & -0.25 & 26.1 & -0.16 \\
\hline & $80 \%$ & 38.47 & 16.20 & 44.35 & 11.00 & 12.39 & 0.22 & $34.8:$ & -0.31 \\
\hline & $100 \%$ & 51.20 & 20.41 & 54.38 & 13.08 & 16.38 & -0.46 & 44.69 & -0.20 \\
\hline & Ave & 29.42 & 12.29 & 32.76 & 7.91 & 9.94 & -0.28 & 26.31 & -0.38 \\
\hline \multirow{6}{*}{ Letter } & $20 \%$ & 5.10 & 2.64 & 3.33 & 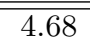 & 0.17 & 2.85 & 4.53 & 2.63 \\
\hline & $40 \%$ & 9.57 & 5.95 & 6.63 & 8.43 & 0.17 & 5.97 & 9.53 & 3.64 \\
\hline & $60 \%$ & 14.12 & 8.67 & 9.18 & 11.59 & 0.17 & 9.36 & 15.47 & 4.54 \\
\hline & $80 \%$ & 18.66 & 11.74 & 13.02 & 15.32 & 0.17 & 12.54 & 21.18 & 6.10 \\
\hline & $100 \%$ & 23.18 & 14.65 & 16.34 & 18.29 & 0.17 & 15.02 & 25.72 & 6.69 \\
\hline & Ave & 14.12 & 8.73 & 9.70 & 11.66 & 0.17 & 9.15 & 15.29 & 4.72 \\
\hline \multirow{6}{*}{ Nursery } & $20 \%$ & 4.97 & 4.59 & 3.80 & 3.12 & 5.52 & 3.78 & 4.85 & 4.38 \\
\hline & $40 \%$ & 9.69 & 9.53 & 9.16 & 8.14 & 13.07 & 8.49 & 9.34 & 9.27 \\
\hline & $60 \%$ & 14.23 & 15.23 & 13.27 & 12.67 & 20.09 & 13.08 & 15.55 & 14.18 \\
\hline & $80 \%$ & 18.93 & 19.60 & 17.90 & 18.23 & 24.57 & 17.86 & 20.33 & 19.45 \\
\hline & $100 \%$ & 23.01 & 25.41 & 22.12 & 22.74 & 30.87 & 21.65 & 24.79 & 25.00 \\
\hline & Ave & 14.16 & 14.87 & 13.25 & 12.98 & 18.82 & 12.97 & 14.98 & 314.46 \\
\hline \multirow{6}{*}{ Connect- 4} & $\overline{20 \%}$ & 4.05 & 0.78 & $\overline{5.08}$ & 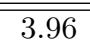 & $\overline{1.54}$ & $\overline{0.78}$ & 8.23 & 0.98 \\
\hline & $40 \%$ & 7.09 & 2.69 & 8.61 & 6.50 & 2.63 & 1.65 & 10.00 & 2.98 \\
\hline & $60 \%$ & 8.47 & 3.48 & 11.98 & 8.93 & 3.85 & 1.79 & 10.54 & 5.03 \\
\hline & $80 \%$ & 12.17 & 5.89 & 14.89 & 13.66 & 6.06 & 2.54 & 13.26 & 7.79 \\
\hline & $100 \%$ & 15.35 & 7.36 & 18.35 & 12.84 & 6.65 & 2.20 & 12.89 & 9.06 \\
\hline & Ave & 9.43 & 4.03 & 11.78 & 9.18 & 4.15 & $\underline{1.79}$ & 10.99 & 5.17 \\
\hline \multirow{6}{*}{ Vote } & $20 \%$ & 7.98 & 7.55 & 0.67 & 8.29 & 6.12 & 0.33 & 6.59 & 8.29 \\
\hline & $40 \%$ & 16.67 & 11.89 & 1.61 & 19.83 & 16.97 & 1.09 & 9.58 & 11.49 \\
\hline & $60 \%$ & 32.15 & 28.82 & 5.05 & 29.72 & 18.61 & 1.12 & 15.16 & 19.80 \\
\hline & $80 \%$ & 40.65 & 30.18 & 3.97 & 35.74 & 32.15 & 3.52 & 17.42 & 27.78 \\
\hline & $100 \%$ & 43.52 & 36.81 & 5.66 & 50.16 & 39.38 & 5.14 & 23.83 & 31.74 \\
\hline & Ave & 28.20 & 23.05 & 3.39 & 28.75 & 22.64 & 2.24 & 14.52 & 19.82 \\
\hline
\end{tabular}

Not surprisingly perhaps, a t-test for a significant difference between the best and the second best classifier does not give us all the information we require. The results are further validated for statistical significance using a one way analysis of variance followed by what is termed a post hoc analysis [15]. First, the learners are tested to see if the average difference in performance, on the original and changed data, of the 8 learners are equal, across the five change levels and the most significant attributes respectively. This is the null hypothesis in ANOVA; the alternative hypothesis is that at least one learner is different. The results of the ANOVA are given in Table 3 and allow us to reject the null hypothesis, but it does not tell us how to rank the classifiers. 
Table 3. ANOVA with their corresponding F-Value and P-Value

\begin{tabular}{|c|c|c|c|c|}
\hline Adult & Letter & Nursery & Connect-4 & Vote \\
\hline F-Value P-Value & F-Value P-Value & F-Value P-Value & F-Value P-Value & F-Value P-Value \\
\hline$<0.0001$ & 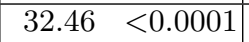 & 0.012 & $11.42<0.0001$ & $25.86<0.0001$ \\
\hline
\end{tabular}

To achieve this we use the post hoc analysis. We apply the Fisher's Least Significant Difference (LSD) test, with an individual error rate of 0.05 . Table 4 provides the average values of each metric for each learner, as well as their significant ranks, the columns labeled Ave and $\mathbf{R}$ respectively in this table. Note that if two or more instances have the same letter, then their performances are not significantly different. Table 4 shows the overall impact of changing the influence of all significant attributes over all change levels. For the Adult data set, the Neural Network and Nearest Neighbor are the most robust learners; the ensemble learners Bagging, AdaBoost and Random Forest are next. For the Letter data set, although AdaBoost is the most robust learner the original accuracy of AdaBoost is close to the default classifier. Excluding AdaBoost from this data set, the Neural Network is the most robust and Random Forest, Nearest Neighbor, Naive Bayes, Bagging are the next. Nearest Neighbor, Random Forests, AdaBoost and the Neural Network are the most robust model, for the Connect4 data set. Next are the Decision Tree, Bagging, Logistic Regression and Naive Bayes. For the Nursery data set, the post hoc test is not able to differentiate among the learners very well. The mean difference value of the learners is close to one another and the variance is high. In this data set all learners except AdaBoost are placed in the first level. For the Vote data set, the best learners are Nearest Neighbor and Naive Bayes, all other learners are in the next group.

Table 4. Overall impact of decreasing attribute influence

\begin{tabular}{|c|c|c|c|c|c|c|c|c|c|c|}
\hline \multicolumn{2}{|c|}{ Adult } & \multicolumn{2}{|c|}{ Letter } & \multicolumn{2}{|c|}{ Connect-4 } & \multicolumn{2}{|c|}{ Nursery } & \multicolumn{2}{|r|}{ Vote } & All data sets \\
\hline Alg & Ave $R$ & $\mathrm{Alg}$ & Ave $R$ & Alg & Ave $R$ & Alg & Ave $R$ & Alg & Ave $R$ & $\begin{array}{lll}\text { Alg } & \text { Ave } & \text { R }\end{array}$ \\
\hline $\mathrm{ANI}$ & $-0.38 \mathrm{~A}$ & $\overline{\mathrm{ADB}}$ & $0.17 \mathrm{~A}$ & KNN & $1.79 \mathrm{~A}$ & KNN & $12.97 \mathrm{~A}$ & KNN & 2.24 & KNN 5.18 A \\
\hline 1 & $\mathrm{~T}-0.28 \mathrm{~A}$ & ANN & $4.72 \mathrm{~B}$ & $\mathrm{RF}$ & $4.03 \mathrm{~A}$ & BG & 12. & NB & $3.39 \mathrm{~A}$ & ANN $8.76 \mathrm{~A}$ \\
\hline$B G$ & 7.91 & $\mathrm{RF}$ & $8.73 \mathrm{C}$ & $\mathrm{ADB}$ & $4.15 \mathrm{~A}$ & NB & 13.2 & LR & $14.52 \mathrm{~B}$ & ADB 1 \\
\hline $\mathrm{ADB}$ & $9.94 \mathrm{~B}$ & KNN & $9.15 \mathrm{C}$ & ANN & $5.17 \mathrm{~A}$ & J48 & 14 . & ANN & N $19.82 \mathrm{~B}$ & $\mathrm{RF} \quad 12.59 \mathrm{~B}$ \\
\hline $\mathrm{RF}$ & $12.29 \mathrm{~B}$ & NB & $9.70 \mathrm{C}$ & $\mathrm{J} 48$ & $9.43 \mathrm{~B}$ & ANN & 14 & $\mathrm{ADB}$ & 322. & $14.1 \mathrm{~B}$ \\
\hline LR & $26.31 \mathrm{C}$ & BG & $11.66 \mathrm{C}$ & $B G$ & $9.18 \mathrm{~B}$ & $\mathrm{RF}$ & $14.87 \mathrm{~A}$ & $\mathrm{RF}$ & $23.05 \mathrm{~B}$ & NB $14.18 \mathrm{~B}$ \\
\hline $\mathrm{J} 48$ & $29.42 \mathrm{C}$ & J48 & $14.12 \mathrm{D}$ & LR & $10.99 \mathrm{~B}$ & LR & $14.98 \mathrm{~A}$ & $\mathrm{~J} 48$ & $28.22 \mathrm{~B}$ & $\mathrm{LR} \quad 16.42 \mathrm{C}$ \\
\hline $\mathrm{NB}$ & $32.76 \mathrm{C}$ & LR & $15.29 \mathrm{D}$ & NB & $11.98 \mathrm{~B}$ & ADB & $18.82 \mathrm{~B}$ & $\mathrm{BG}$ & $28.75 \mathrm{~B}$ & $\mathrm{~J} 48 \quad 19.07 \mathrm{D}$ \\
\hline
\end{tabular}


From table 4, due to the high variance among learners in each data set, the post hoc tests does not differentiate among the learners very well. For the Adult data set, the post hoc test does not differentiate among the learners of the third group indicated by letter C. For the Letter data set, it also does not differentiate among the third group of the learners. This is repeated across the individual data sets.

To improve differentiation of the robustness of algorithms, in the last column of Table 4, we give the average values for each learner, and significant ranks, for all data sets combined. Here, Nearest Neighbor is the clear winner with the Neural Network in second place. Learners reliant on a smaller number of attributes such as Random Forests, AdaBoost and Bagging are next. AdaBoost is the best but, as it did poorly in terms of accuracy on a couple of original data sets, this robustness comes at some price. The Decision Tree comes firmly in last place. In general, our experimental results show that learners reliant on more attributes, tend to be more robust to the changes of the influence of some of them.

\section{Discussion and Future Work}

In this section, we discuss some hypotheses for why some learners are more robust to changing the influence of attributes than others. These need verification and will be the subject of future work. We will also discuss other directions for future research.

For Artificial Neural Networks robustness to attribute changes is dependent on a decision surface using all attributes of the data simultaneously. Thus, the changing of one attribute of an instance is unlikely to cause that instance to be misclassified, unless the weight of that attribute is very much larger than that of others. Likewise, for Nearest Neighbor all attributes are used to find the distance between two instances. If the influence of one attribute decreases, other attributes can still be predictive. For decision trees such as J48, where the complexity of the tree depends on the data set we use, often only a few attributes define the decision boundary. Thus, if the influence of one of these attributes on one particular instance on the class is changed, then that instance may well cross the decision boundary.

For example, Figure 1(a) is the decision tree and Figure 1(b) is the data and decision boundary formed by the tree (solid lines) and that formed by the Neural Network (dashed line). Note that $\mathrm{x}$ is the most significant attribute for decision tree. Now suppose that by changing the influence of this attribute, the instance at the top of Figure 1 moves in the $\mathrm{x}$ direction crossing the decision boundary for the tree. That instance will now travel down the right branch of the decision tree as shown by the dashed line in Figure 1(a), classifying this instance incorrectly. This instance does not cross the decision boundary for the Neural Network. This is because this boundary uses both attributes and is much smoother. The same reasoning can be applied to the instance that crosses the $\mathrm{y}=\mathrm{c}$ boundary in Figure 1 (b). If by changing $y$, the second most influential attribute, the instance crosses 


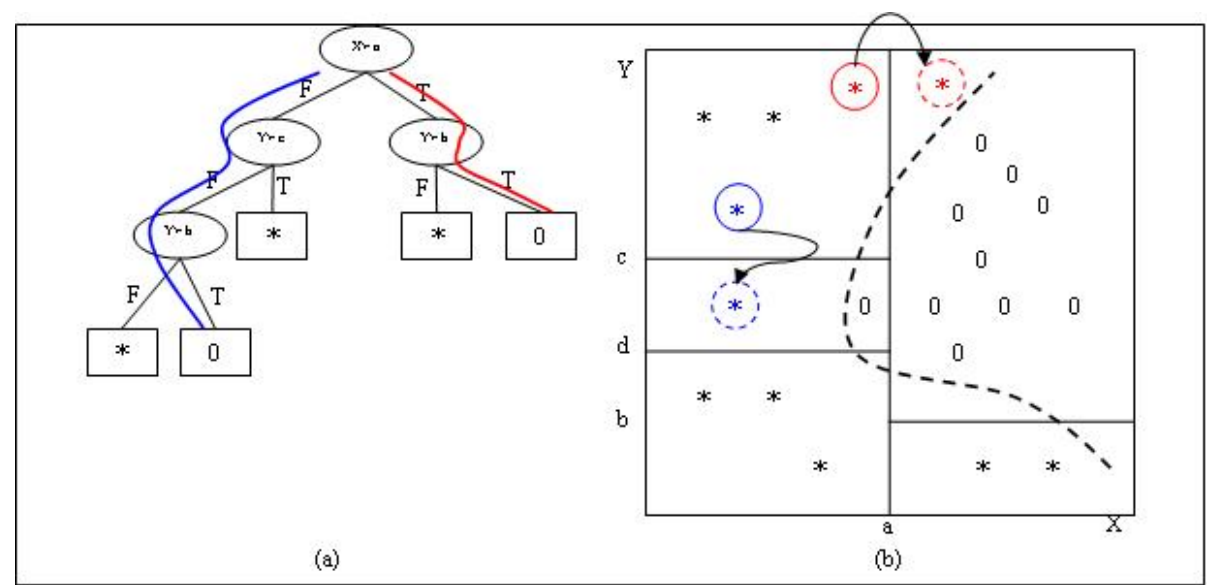

Fig. 1. The impact of noise on decision tree and Artificial neural network

the boundary $\mathrm{y}=\mathrm{c}$ then the left solid branch on the tree classifies this instance as 0 but the Neural Network correctly classifies it.

Ensemble algorithms, as they consist of multiple classifiers, tend to rely on many, though not all, attributes. Where they differ, from each other, is primarily in the way the individual classifiers are constructed. We expect that Random Forests, by deliberately selecting classifiers based on different attributes, would be the most robust. That is supported by our experimental results in that it does well on a couple of data sets. It is, however, beaten overall by AdaBoost, although the poor performance of AdaBoost on some original data sets accounts for much of this. Further experiments are needed to determine whether or not there is any real difference between ensemble algorithms in terms of robustness. If there is, we aim to find out what is at the root these differences. What is clear, however, is that they are generally better than the base classifier they use.

We had concerns that the statistical tests, which showed AdaBoost to be very robust, might be misleading due to its poor performance on some of the original data sets. So, we claim that for an algorithm to be considered robust it must not only have a small difference in performance, but also the performance on the original data set must be good, or at least competitive with other algorithms. As future work, it would be worth exploring if a single metric might capture these two concerns. An alternative would be to plot points showing how they trade-off on a two-dimensional graph, similar to ROC curve or cost curves $[9,5]$. Another concern with the experiments is that the measure we used, information gain ratio, is also used by the decision tree algorithm to chose the attributes at each branch. So, it may have a larger impact on the performance of decision trees than on that of other algorithms. We will therefore explore the effect of using different measures for selecting the most significant attributes in the future experiments. This can be quite easily realized within Weka as it has many attribute evaluators: Info Gain, Relief Attribute Evaluator, Principal Components, and OneR. 
As other future work, the experiments will look at a greater range of algorithms. It would be worth exploring if there are other general characteristics, apart from the number of attributes used, that affect robustness. Another simple extension of current research is to include more data sets. We will also use the entire number of instances of the present data sets instead of just a $10 \%$ fraction of them. Some of the data sets we chose only had one significant attribute. This property has been noticed before, simple classifiers often do well on UCI data sets [6]. It would be worth doing experiments on data sets with a wide spread in the number of significant attributes, to see how this affects robustness. We will also experiment with changing the influence of a combination of attributes instead of one attribute at a time and with changing attributes in other ways.

We believe this paper has given some insight into what makes a classifier robust to changing environments. Nevertheless we have not explained by any means all the factors. We need to determine why Naive Bayes and Logistic Regression, which use all attributes, are not robust. Further experiments will be needed to expose the other differences between classifiers.

\section{Conclusions}

The objective of this study was to investigate the robustness of a variety of commonly used learning algorithms to changing environments. The Neural Network and Nearest Neighbor are the most robust, learners reliant on smaller number of attributes such as Random Forest, AdaBoost and Bagging are located in the second place and finally the Decision Tree is in last place. In general, we conclude that learners reliant on more attributes tend to be more robust. This is clearly not the whole story, however, as Naive Bayes and Logistic Regression are not robust, future work will investigate this issue further.

\section{Acknowledgments}

We would like to thank the Natural Sciences and Engineering Research Council of Canada for the financial support that made this research possible.

\section{References}

1. R. Alaiz-Rodríguez, A. Guerrero-Curieses, and J. Cid-Sueiro. Minimax regret classifier for imprecise class distributions. Journal of Machine Learning Research, 8:103-130, 2007.

2. R. Alaiz-Rodríguez and N. Japkowicz. Assessing the impact of changing environments on classifier performance. In Proceedings of the 21st Canadian Conference in Artificial Intelligence, pages 13-24, 2008.

3. Cathy Blake and Christopher Merz. UCI repository of machine learning databases. univ. of california at irvine. http://www.ics.uci.edu/ mlearn/MLRepository . html, 1998. 
4. Chris Drummond. Discriminative vs. generative classifiers for cost sensitive learning. In L. Lamontagne and M. Marchand, editors, Proceedings of the Nineteenth Canadian Conference on Artificial Intelligence, LNAI 4013, pages 481-492, 2006.

5. Chris Drummond and Robert C. Holte. Cost curves: An improved method for visualizing classifier performance. Machine Learning, 65(1):95-130, 2006.

6. Robert C. Holte. Very simple classification rules perform well on most commonly used datasets. Machine Learning, 11(1):63-91, 1993.

7. J. Huang, A.J. Smola, A.G retton, K.M. Borgwardt, and B. Schölkopf. Correcting sample selection bias by unlabeled data. In B. Schölkopf, J.Platt, and T.Hoffman, editors, Advances in Neural Information Processing Systems 19, pages 601-608. MIT Press, 2007.

8. F. Provost and T. Fawcett. Robust classification for imprecise environments. $M a-$ chine Learning, 42:203-231, 2001.

9. Foster Provost, Tom Fawcett, and Ron Kohavi. The case against accuracy estimation for comparing induction algorithms. In Proceedings of the Fifteenth International Conference on Machine Learning, pages 43-48, San Francisco, 1998. Morgan Kaufmann.

10. J. Ross. Quinlan. C4.5 Programs for Machine Learning. Morgan Kaufmann, San Mateo, California, 1993.

11. Joaquin Quionero-Candela, Masashi Sugiyama, Anton Schwaighofer, and Neil D. Lawrence. Dataset Shift in Machine Learning. The MIT Press, 2009.

12. P.D. Turney. Exploiting context when learning to classify. In Proceedings of the European Conference on Machine Learning, pages 402-407, 1993.

13. P.D. Turney. The management of context-sensitive features: A review of strategies,. In Proceedings of the 13th International Conference on Machine Learning: Workshop on Learning in Context-Sensitive Domains, pages 60-66, 1996.

14. G. Widmer and M. Kubat. Learning in the presence of concept drift and hidden contexts. Machine Learning, 23:69-101, 1996.

15. wikipedia. http://en.wikipedia.org/wiki/Post-hoc_analysis.

16. Ian H. Witten and Eibe Frank. Data Mining: Practical machine learning tools and techniques. Morgan Kaufmann, 2005. 